Article

\title{
Comparison of Correlates of Bone Mineral Density in Individuals Adhering to Lacto-Ovo, Vegan, or Omnivore Diets: A Cross-Sectional Investigation
}

\section{Jessica R. Knurick ${ }^{1}$, Carol S. Johnston ${ }^{1, *}$, Sarah J. Wherry ${ }^{2}$ and Izayadeth Aguayo ${ }^{3}$}

1 School of Nutrition and Health Promotion, Arizona State University, 500 N. 3rd Street, Phoenix, AZ 85004, USA; E-Mail: jessica.knurick@asu.edu

2 Department of Medicine, University of Colorado, 12631 East 17th Avenue, Aurora, CO 80045, USA; E-Mail: sarah.wherry@ucdenver.edu

3 The University of Arizona College of Medicine-Phoenix, 550 E. Van Buren St., Phoenix, AZ 85004, USA; E-Mail: iaguayo@email.arizona.edu

* Author to whom correspondence should be addressed; E-Mail: carol.johnstona@asu.edu; Tel.: +1-602-827-2265; Fax: +1-602-827-2253.

Received: 13 February 2015 / Accepted: 13 April 2015 / Published: 11 May 2015

\begin{abstract}
Vegetarian diets are associated with factors that may not support bone health, such as low body mass and low intakes of protein; yet, these diets are alkaline, a factor that favors bone mineral density (BMD). This study compared the correlates of BMD in young, non-obese adults consuming meat-based $(n=27)$, lacto-ovo vegetarian $(n=27)$, or vegan ( $n=28$ ) diets for $\geq 1$ year. A $24 \mathrm{~h}$ diet recall, whole body DXA scan, $24 \mathrm{~h}$ urine specimen, and fasting blood sample were collected from participants. BMD did not differ significantly between groups. Protein intake was reduced $\sim 30 \%$ in individuals consuming lacto-ovo and vegan diets as compared to those consuming meat-based diets (68 \pm 24 , $69 \pm 29$, and $97 \pm 47$ g/day respectively, $p=0.006$ ); yet dietary protein was only associated with BMD for those following vegan diets. Urinary $\mathrm{pH}$ was more alkaline in the lacto-ovo and vegan groups versus omnivores $(6.5 \pm 0.4,6.7 \pm 0.4$, and $6.2 \pm 0.4$ respectively, $p=0.003$ ); yet urinary $\mathrm{pH}$ was associated with BMD in omnivores only. These data suggest that plant-based diets are not detrimental to bone in young adults. Moreover, diet prescriptions for bone health may vary among diet groups: increased fruit and vegetable intake for individuals with high meat intakes and increased plant protein intake for individuals who follow a vegetarian diet plan.
\end{abstract}


Keywords: vegetarian; vegan; bone mineral density; protein; acid-base balance

\section{Introduction}

The adoption of a vegetarian diet has become increasingly popular in light of expert recommendations to follow plant-based diets for improved health outcomes. Current estimates suggest that between $3 \%$ and $5 \%$ of the U.S. population follows some variation of a vegetarian diet [1]. Vegetarianism is associated with health benefits including lower rates of obesity, diabetes, hypertension, cardiovascular disease, and some cancers [2,3]. However, the restrictive nature of the diet has prompted concern regarding possible nutrient deficiencies and a heightened risk for osteoporosis [4].

Bone is a dynamic and metabolically active tissue that requires adequate nutrients for bone modeling and mineralization throughout the life cycle. While vegetarianism is associated with several factors that may not support bone health, such as low body mass index (BMI) and low intakes of calcium, vitamins D and B12, and protein, these diets are high in nutrients that promote bone health, including magnesium, potassium, vitamins $\mathrm{C}$ and $\mathrm{K}$, and the $n-3$ fatty acids [5,6]. Vegetarian diets are also more alkaline than omnivorous diets, a factor that favors higher bone mineral density [7-9]. Furthermore, the concern that vegetarian diets may adversely affect bone mineral density (BMD) and fracture risk may be conjectural as findings from several prospective longitudinal investigations did not reveal harmful effects of vegetarianism on bone health [10,11]. A 2009 meta-analysis (nine studies totaling 2749 subjects of which 68\% were female) concluded that BMD was $4 \%$ lower in vegetarians as compared to non-vegetarians [12]. The authors inferred that fracture risk was $\sim 10 \%$ greater for vegetarians versus non-vegetarians but concluded that this risk was clinically insignificant [12].

Hence, it is possible that bone-healthy components of vegetarian diets counterbalance the negative effects of a low BMI and low intakes of protein and calcium. The aim of this study was to investigate the associations between indicators of bone health and BMD in young, healthy, non-obese, sedentary adults adhering to meat-based, lacto-ovo vegetarian, or vegan diet.

\section{Experimental Section}

\subsection{Participants and Diet Classification}

Nonsmoking participants were recruited through university list serves, local vegetarian societies, and word-of-mouth. Based on responses to an online survey, volunteers were recruited using the following criteria: age 19-50 year, body mass index (BMI; in $\mathrm{kg} / \mathrm{m}^{2}$ ) $<30$, respective diet adherence $>1$ year, and absence of any known chronic diseases. Additionally, volunteers were excluded if they were competitive athletes or training for an endurance event, were taking prescribed medications (other than oral contraception), and/or reported (if female) an irregular menstrual cycle or history of pregnancy within the preceding six months. The study was approved by the Arizona State University Institutional Review Board, and all participants provided written informed consent. 
The classification of diet group and duration of adherence was assessed by the following four questions: (1) "How many times per week do you consume meat, fish, or poultry?” (2) "How many times per week do you consume dairy products (milk, cheese, butter, yogurt)?” (3) How many eggs do you consume per week?” (4) “How long have you been following your current diet?” Subjects who reported consuming at least three servings of meat, fish, or poultry per week were considered omnivores. Those who consumed between zero and three servings were excluded from the study. Individuals who reported never eating meat, fish, or poultry, but consumed at least three servings of dairy and/or three eggs per week were considered lacto-ovo vegetarians. Individuals who reported never consuming meat, fish, or dairy and never consuming dairy or eggs were considered vegan. All subjects reported following their current diet for at least one year. Physical activity levels were estimated by validated questionnaire and reported as metabolic equivalents (METS) [13].

\subsection{Study Design}

Participants visited the test site on two occasions. At the first visit, subjects completed a questionnaire that included details on age, gender, smoking status, medical history, and activity level. A 24 h diet recall was conducted by a trained nutritionist who also completed the nutritional analysis (Food Processor version 7.71; ESHA Research, Salem, OR). Fortified food products commonly consumed by vegetarian populations were manually entered if necessary to be properly accounted in the diet analyses. Diet quality was assessed by the validated REAPS scoring system [14]. Potential renal acid load (PRAL) was estimated from dietary intake using the equation: (0.49 protein (g)) + $(0.037 \mathrm{P}(\mathrm{mg}))-(0.021 \mathrm{~K}(\mathrm{mg}))-(0.026 \cdot \mathrm{Mg}(\mathrm{mg}))-(0.013 \mathrm{Ca}$ (mg)) [15]. Anthropometric measurements including height, body weight, and waist circumference were obtained, as well as body fat percentage and total body bone mineral density using dual-energy X-ray absorptiometry (DXA; Lunar iDXA, General Electric Company, East Cleveland, OH, USA). Participants collected a $24 \mathrm{~h}$ urine sample the day prior to the second visit using the following instructions: discard the first morning void but collect all subsequent urine voids for the day including the first urine void of the second morning. Participants were provided with urine containers and collection vessels.

Participants returned to the test site for the second visit within one week in a fasted state (no food or beverage with the exception of water for $12 \mathrm{~h}$ ). Urine samples were turned in, and a venous blood sample was collected. Urinary calcium, measured photometrically, and the blood anion gap were measured at Sonora Quest Laboratories (Tempe, AZ, USA). Urinary $\mathrm{pH}$ was determined using a $\mathrm{pH}$ meter (WTW Chekmite pH-20 Sensor, Nova pH, Woburn, MA, USA).

\subsection{Statistical Analyses}

Data are reported as mean $\pm \mathrm{SD}$; statistical analyses were performed using SPSS for WINDOWS (version 21; SPSS Inc., Chicago, IL, USA). Differences between means were assessed using univariate analyses controlling for age, which differed significantly between diet groups, BMI, and gender. Non-normal data were transformed prior to analysis, and, if transformation did not normalize the data, the Kruskal Wallis test was used. Nominal data were compared using Chi Square analysis. Pearson's correlation was used to identify relations between variables controlling for age, BMI, and gender. Significance was set at $p \leq 0.05$. 


\section{Results}

A total of 409 individuals completed the online survey, and 82 qualifying individuals agreed to enroll in the study. Based on self-reported diet habits, participants were grouped into three diet groups: omnivore ( $n=27)$, lacto-ovo vegetarian $(n=27)$, and vegan $(n=28)$. Participant retention was $100 \%$; however, one participant in the lacto-ovo vegetarian group did not complete the $24 \mathrm{~h}$ diet recall.

Individuals adhering to a vegan diet were significantly older than individuals adhering to a meat-based diet (33.9 \pm 8.6 year versus $27.2 \pm 6.7$ year respectively); whereas, the mean age of lacto-ovo vegetarians did not vary significantly from the other diet groups (31.1 \pm 9.1 year) (Table 1). Anthropometric measures and physical activity levels did not differ significantly between diet groups, but BMI tended to be higher for individuals adhering to a meat-based diet as compared to individuals adhering to lacto-ovo or vegan diets (23.5 \pm 3.1 (range, 18.7-29.0), $22.4 \pm 2.7$ (range, 18.3-28.1), and $22.3 \pm 2.6$ (range, 18.9-28.0) kg/m² respectively, $p=0.092$ ). Diet quality was superior for individuals adhering to a vegan diet as compared to the other diet groups (Table 1).

Table 1. Participant characteristics by diet group (omnivorous, OMN; lacto-ovo vegetarian, LOV; vegan, VEGAN) ${ }^{1}$.

\begin{tabular}{|c|c|c|c|c|}
\hline & OMN & LOV & VEGAN & $p$ \\
\hline Gender, M/F (\%F) & 8/19 (70) & 6/21 (78) & $10 / 18(64)$ & 0.546 \\
\hline Age, year & $27.2 \pm 6.7^{\mathrm{a}}$ & $31.1 \pm 9.1^{\mathrm{a}, \mathrm{b}}$ & $33.9 \pm 8.6^{\mathrm{b}}$ & 0.013 \\
\hline Body weight, Kg & $66.8 \pm 12.0$ & $62.9 \pm 10.0$ & $64.6 \pm 12.0$ & 0.218 \\
\hline Height, cm & $168.0 \pm 10.5$ & $167.1 \pm 7.4$ & $169.3 \pm 8.9$ & 0.716 \\
\hline BMI, $\mathrm{Kg} / \mathrm{m}^{2}$ & $23.5 \pm 3.1$ & $22.4 \pm 2.7$ & $22.3 \pm 2.6$ & 0.092 \\
\hline Waist, cm & $80.5 \pm 10.6$ & $79.8 \pm 9.8$ & $80.1 \pm 9.6$ & 0.615 \\
\hline Body fat,\% & $29.1 \pm 8.8$ & $31.7 \pm 7.6$ & $28.8 \pm 7.3$ & 0.269 \\
\hline Visceral fat, $\mathrm{cm}^{3}$ & $246.0 \pm 238.0$ & $254.1 \pm 298.2$ & $435.7 \pm 428.4$ & 0.260 \\
\hline METS, kcal kg ${ }^{-1}$ week $^{-1}$ & $50.2 \pm 39.0$ & $37.4 \pm 28.8$ & $40.1 \pm 27.5$ & 0.569 \\
\hline Diet quality, REAPS score & $31.8 \pm 3.2^{\mathrm{a}}$ & $32.4 \pm 2.7^{\mathrm{a}}$ & $36.1 \pm 2.0^{\mathrm{b}}$ & $<0.001$ \\
\hline
\end{tabular}

BMD was reduced $4 \%-5 \%$ in individuals adhering to meatless diets as compared to the omnivores; however, this difference was not significant (Table 2). Z scores did not differ significantly between diet groups and placed BMD between the 75th and 80th percentiles for meat-eaters and between the 60th and 70th percentiles for vegetarians. Calcium excretion (mg/24 h) was significantly higher $(\sim 34 \%)$ in the omnivores as compared to individuals adhering to vegetarian diets $(p=0.045)$. Several acid-base indices (urinary $\mathrm{pH}$ and dietary $\mathrm{PRAL}$ ) varied significantly between diet groups. Urinary $\mathrm{pH}$ was more alkaline in the vegetarian diet groups versus the omnivores $(6.5 \pm 0.4$ and $6.7 \pm 0.4$ for the lacto-ovo and vegan groups versus $6.2 \pm 0.4$ for the omnivores, $p=0.003$ ). Dietary PRAL was reduced over $100 \%$ in the vegetarian groups as compared to the omnivores ( $p=0.001$; Table 2$)$. PRAL was significantly related to urinary $\mathrm{pH}$ among all participants $(r=-0.322, p=0.004)$.

The intake of several nutrients commonly associated with bone health varied by diet group (Table 3). Protein intake was reduced $\sim 30 \%$ in individuals adhering to vegetarian diets as compared to 
those consuming a meat-based diet (68 \pm 24 and $69 \pm 29 \mathrm{~g}$ /day for individuals adhering to lacto-ovo or vegan diets versus $97 \pm 47 \mathrm{~g}$ /day for individuals eating a meat-based diet, $p=0.006)$. Additionally, individuals adhering to vegan diets consumed significantly more magnesium, folate, and vitamin $\mathrm{K}$ as compared to their omnivorous counterparts (Table 3). However, vitamin B12 intakes were significantly reduced in the vegetarian diets versus the meat-based diet. Dietary protein was significantly correlated with both BMD and urinary calcium among all participants $(r=0.274$, $p=0.017$ and $r=0.228, p=0.049$ respectively). However, when examined by diet group, dietary protein was significantly related to BMD only in the vegan diet group (Table 4); moreover, dietary protein was not associated with urinary calcium in any single diet group. No other significant associations were noted for individual nutrients and BMD for any diet group. Urinary pH was the only other variable significantly related to BMD when examined by diet group, an association only noted in the omnivore diet group ( $r=0.602, p=0.002$; Table 4). There was no association between urinary $\mathrm{pH}$ and BMD in the sample as a whole.

Table 2. Bone mineral density and urinary measures by diet group (omnivorous, OMN; lacto-ovo vegetarian, LOV; vegan, VEGAN) ${ }^{1}$.

\begin{tabular}{ccccc}
\hline & OMN $(\boldsymbol{n}=\mathbf{2 7})$ & LOV $(\boldsymbol{n}=\mathbf{2 7})$ & VEGAN $(\boldsymbol{n}=\mathbf{2 8})$ & $\boldsymbol{p}$ \\
\hline Bone mineral density, g/cm & & \\
$T$ score & $1.18 \pm 0.11$ & $1.12 \pm 0.10$ & $1.13 \pm 0.11$ & 0.384 \\
$Z$ score & $0.71 \pm 0.97$ & $0.13 \pm 0.93$ & $0.03 \pm 0.85$ & 0.286 \\
Urinary calcium, mg/24h & $0.77 \pm 0.77$ & $0.42 \pm 0.75$ & $0.26 \pm 0.86$ & 0.337 \\
Anion Gap & $155 \pm 71^{\mathrm{a}}$ & $115 \pm 64^{\mathrm{b}}$ & $117 \pm 65^{\mathrm{b}}$ & 0.045 \\
Urinary pH & $12.5 \pm 2.6$ & $11.9 \pm 2.0$ & $11.9 \pm 2.4$ & 0.562 \\
PRAL & $6.2 \pm 0.4^{\mathrm{a}}$ & $6.5 \pm 0.4^{\mathrm{b}}$ & $6.7 \pm 0.4^{\mathrm{b}}$ & 0.003 \\
\hline
\end{tabular}

\footnotetext{
${ }^{1} p$ for univariate analyses analysis (non-normal data transformed prior to analysis (urinary calcium, anion gap)); all $p$ values adjusted for BMI, age, and gender; means with different superscripts differ significantly $p<0.05$. Sample reduced for PRAL due to a missing diet recall ( $n=25,26$, and 28 for OMN, LOV and VEGAN respectively). ${ }^{2}$ Non parametric Kruskal-Wallis test.
}

\section{Discussion}

Bone is sensitive to its environment and slight changes in nutrient availability and acid-base balance have acute effects on bone metabolism, which in the long term would theoretically impact BMD [16,17]. BMD did not differ between lacto-ovo vegetarians, vegans, and omnivores in the present study supporting the contention that plant-based diets are not detrimental to bone in young adults. Vegetarian diets, particularly vegan diets, have a nutrient profile that varies significantly from meat-based diets, and many of the nutrient differences could theoretically impact bone metabolism, some favorably and some unfavorably. In young adults, a well-balanced vegetarian diet may provide adequate bone-enhancing properties that negate the diet properties that adversely impact bone mineralization. However, these data cannot provide insights regarding the impact of vegetarian diets on bone health in older adults or the elderly. 
Table 3. Dietary intake for nutrients associated with bone health by diet group (omnivorous, OMN; lacto-ovo vegetarian, LOV; vegan, VEGAN) ${ }^{1}$.

\begin{tabular}{|c|c|c|c|c|}
\hline & OMN $(n=27)$ & $\operatorname{LOV}(n=26)$ & VEGAN $(n=28)$ & $\boldsymbol{P}$ \\
\hline Energy, kcal & $2108 \pm 727$ & $2042 \pm 558$ & $2069 \pm 665$ & 0.856 \\
\hline Protein, g & $97 \pm 47^{\mathrm{a}}$ & $68 \pm 24^{b}$ & $69 \pm 29^{b}$ & 0.006 \\
\hline Calcium, mg & $939 \pm 516$ & $746 \pm 422$ & $768 \pm 415$ & 0.241 \\
\hline Magnesium, mg & $210 \pm 144^{\mathrm{a}}$ & $241 \pm 135^{\mathrm{a}}$ & $354 \pm 183^{b}$ & 0.006 \\
\hline Potassium, mg & $2048 \pm 1153$ & $2135 \pm 1220$ & $2876 \pm 1934$ & 0.154 \\
\hline Sodium, mg & $3743 \pm 1877$ & $2871 \pm 1389$ & $2522 \pm 1228$ & 0.067 \\
\hline Zinc, mg & $8.7 \pm 8.2$ & $5.6 \pm 3.8$ & $8.5 \pm 6.1$ & 0.210 \\
\hline Folate, ug & $286 \pm 233^{a}$ & $391 \pm 290^{a b}$ & $549 \pm 367^{b}$ & 0.006 \\
\hline Vitamin B6, mg & $1.5 \pm 1.0$ & $2.5 \pm 4.2$ & $2.4 \pm 2.2$ & $0.256^{2}$ \\
\hline Vitamin B12, mg & $4.9 \pm 8.0^{\mathrm{a}}$ & $2.3 \pm 3.4^{b}$ & $3.3 \pm 5.1^{b}$ & $0.051^{2}$ \\
\hline Vitamin C, mg & $149 \pm 134$ & $140 \pm 128$ & $225 \pm 177$ & 0.143 \\
\hline Vitamin D, ug & $2.2 \pm 2.9$ & $1.3 \pm 2.5$ & $1.7 \pm 2.3$ & $0.089^{2}$ \\
\hline Vitamin K, ug & $100 \pm 200^{a}$ & $159 \pm 251^{a b}$ & $557 \pm 871^{b}$ & 0.015 \\
\hline$n-6$ fatty acids, $g$ & $6.3 \pm 7.4$ & $5.8 \pm 5.8$ & $4.0 \pm 3.1$ & $0.479^{2}$ \\
\hline$n-3$ fatty acids, $g$ & $0.8 \pm 0.8$ & $0.7 \pm 1.0$ & $1.3 \pm 2.2$ & $0.626^{2}$ \\
\hline$n-6 / n-3$ ratio & $8.8 \pm 5.3^{a b}$ & $10.8 \pm 7.3^{a}$ & $6.4 \pm 5.4^{b}$ & 0.026 \\
\hline
\end{tabular}

${ }^{1} p$ for univariate analyses analysis (transformed prior to analysis: protein, calcium, magnesium, potassium, sodium, zinc, folate, vitamin K, $n-6$ and $n-3$ fatty acids); all $p$ values adjusted for BMI, age, and gender; means with different superscripts differ significantly $p<0.05 .{ }^{2}$ Non parametric Kruskal-Wallis test.

Table 4. Correlation coefficients for urinary $\mathrm{pH}$ and protein significantly correlated with $\mathrm{BMD}$ in at least one diet group ${ }^{1}$.

\begin{tabular}{cccc}
\hline & OMN & LOV & VEGAN \\
\hline Urinary pH & $0.602 *$ & 0.003 & -0.008 \\
Protein, g & 0.190 & 0.262 & $0.434 *$ \\
\hline${ }^{1}$ Asterisk denotes a significant correlation: Pearson's Correlation, $p<0.05$.
\end{tabular}

Much evidence demonstrates the importance of dietary protein for bone accrual and reduced fracture risk particularly in older adults; hence, this nutrient is of particular concern since vegetarian diets are comparatively low in protein. The anabolic influence of protein on bone is mediated by insulin-like growth factor-1, which increases plasma osteocalcin and promotes osteoblast recruitment and activity [18]. Elderly patients with a recent osteoporotic hip fracture ingesting a protein supplement (20 g/day) experienced marked elevations in insulin-like growth factor-1 (+84\%) and significant attenuation of proximal femur bone loss as compared to their control counterparts after six months [19]. Dietary protein also promotes bone mineralization by enhancing calcium absorption rates; however, adequate levels of dietary calcium ( $\geq 1000 \mathrm{mg} /$ day) are likely necessary for this effect [20]. A cohort study based in California with 1865 female participants ( $\geq 45$ year at study start) and a 25 year follow-up reported a significantly higher incidence of wrist fracture in vegetarians as compared to omnivores and that higher intakes of vegetable protein by vegetarians, from $<3 \times$ weekly to $>1 \times$ daily, reduced this risk by $68 \%$ [10]. Conversely, the incidence of vertebral fracture risk did not differ between vegetarians and omnivores in an Asian cohort of postmenopausal women followed for two years (incidence of fracture: 5.7\% and 5.4\% respectively), even though vegetarian participants consumed $40 \%$ less protein daily than their omnivore counterparts [11]. However, the daily protein 
intake was relatively low even for the omnivores (62 g/day), and the rates of fracture were much higher than that reported for the California cohort (3.7/1000 person-years). A two year prospective, cohort trial in female collegiate runners ( $22 \pm 3$ years) did report a significant, positive impact of animal protein intake on bone accrual, a relationship that remained significant after controlling for calcium intake [21]. However, in this study, dietary protein was not related to fracture risk.

Although important for bone anabolism, a high protein intake can adversely impact bone health as it contributes to the dietary acid load, a consequence of the hydrogen ions produced during the oxidation of the sulfur-amino acids, methionine and cysteine [22]. Since the dietary acid load stimulates osteoclast activity and bone resorption, enabling the release of neutralizing carbonate and hydroxyapatite salts from bone, bone mineralization is hindered [23,24]. The dietary acid load is estimated by measuring $24 \mathrm{~h}$ urinary $\mathrm{pH}$, an indirect marker of net acid excretion [25], or from daily intakes of protein and phosphorus, and the alkaline salts, calcium, potassium, and magnesium, using a formula known as PRAL, the potential renal acid load [15].

Demonstrating the two-edged effect of dietary protein on bone, Thorpe and colleagues reported that although increasing dietary protein was associated with higher BMD in postmenopausal women, this favorable effect was attenuated by a high sulfur intake [26]. These results may help explain the lack of an association between dietary protein and BMD in omnivores in the present study. The inter-quartile range for dietary protein in the omnivores (69-124 g) was above the recommended protein intake for this participant sample (52 g; calculated using the U.S. Food and Nutrition Board's dietary goal: $0.8 \mathrm{~g} / \mathrm{kg}$ body weight). High intakes of protein likely contributed to the dietary acid load as indicated by the low urinary $\mathrm{pH}$ for omnivores; moreover, urinary $\mathrm{pH}$ predicted $36 \%$ of the variance in BMD in this diet group. The inter-quartile range of dietary protein in the lacto-ovo and vegan diet groups, 44-93 g and 51-87 g respectively, encompassed the dietary goal for protein, and dietary protein was not related to urinary $\mathrm{pH}$ in these diet groups. However, dietary protein was strongly related to BMD in the vegan diet group suggesting the importance of ample dietary protein for bone health even in a marked alkaline environment (urinary pH, $6.7 \pm 0.4$; PRAL, $-15.2 \pm 40.5$ ).

High intakes of fruits and vegetables reduce the dietary acid load by providing neutralizing anions, e.g., citrate and malate, found mainly in association with potassium and magnesium cations, and glutamate, a neutralizing anionic amino acid [27]. Large controlled trials have demonstrated the benefits of supplemental potassium citrate on BMD in postmenopausal women with osteopenia after one year [28] and in healthy elderly men and women after two years [29]; however, Macdonald et al. [30] were unable to increase BMD in healthy postmenopausal women with potassium citrate supplements taken daily for two years. Plant-based diets are also high in vitamin K, an essential cofactor in the carboxylation of osteocalcin necessary for optimal mineralization of bone [31]. Vegan diets contained at least $35 \%, 47 \%$, and $250 \%$ more potassium, magnesium, and vitamin $\mathrm{K}$ respectively than the other diet plans, a characteristic reflected by the very low PRAL score and high urinary $\mathrm{pH}$ of the vegan diet group. The similar BMD among the diet groups may reflect a cancelling effect occurring with the different diet plans: high dietary protein (bone positive) with high dietary acid load (bone negative) in the omnivore group but low dietary protein (bone negative) with low dietary acid load (bone positive) for the vegetarian groups. A healthy bone diet prescription may be to increase fruit and vegetable intake among individuals with high meat intakes and to increase plant protein intake among individuals who follow a vegetarian diet plan. 
Gunn and colleagues [32] examined the feasibility of increasing fruit and vegetable consumption in 21 healthy omnivorous adult women (40-65 year of age; typical fruit and vegetable intake $<5$ servings/day) in order to reduce the renal acid load as indicated by an increase in urinary $\mathrm{pH}$. After the 8-week intervention, participants increased fruit consumption by 1.4 servings/day and vegetable consumption by 2.3 servings/day; a non-significant reduction in dietary protein was also noted (80 $\mathrm{g}$ at baseline and $73 \mathrm{~g}$ at week $8 ; p=0.29$ ). Importantly, urinary $\mathrm{pH}$ increased significantly, from 5.46 at baseline to 6.14 at week 8 . Intervention studies are needed to determine if a change in acid-base balance due to increased fruit and vegetable consumption favors a bone formation environment in omnivores. To date, the effect of increased protein intakes on bone health in vegetarians have not been conducted; yet, the results from several intervention trials suggest a benefit of supplemental soy protein for improving muscle mass and strength in older men consuming a vegetarian diet plan during 12 weeks of resistive training [33,34]. However, care may be needed when recommending higher protein intakes for vegetarians since some popular vegetarian protein sources, e.g., hard cheeses, eggs, brown rice, and rolled oats, have PRAL scores similar to, or greater than, lean beef and turkey $(\geq 7.8)[35]$.

\section{Limitations of the Study}

The results of this trial only suggest possible influences of omnivorous and vegetarian diet plans on BMD due to the cross-sectional study design and to the small sample sizes by diet group. Also, the placement of participants into diet groups based on self-reported dietary intakes is a major study limitation as are the narrow age range of participants and the lack of site-specific measures of BMD. Single-day diet analyses cannot account for the daily intraindividual variation in food intake over time, which limits dietary data interpretation. However, the $24 \mathrm{~h}$ diet recalls were performed by a trained nutritionist and carefully conducted as indicated by energy intakes that matched energy expenditure prediction equations within a 10\% margin. Similarly, a random $24 \mathrm{~h}$ urine collection may not capture daily intraindividual variation in $\mathrm{pH}$, but a $24 \mathrm{~h}$ urine sample, as compared to a fasting urine sample, will control for the diurnal variation in urine $\mathrm{pH}$ [36]. Trial strengths include the $100 \%$ retention rate, and the use of DXA for total body BMD determination and 24-h urine samples for the measurement of urinary $\mathrm{pH}$. Participants were also carefully selected to reduce confounding by known predictors of BMD: physical activity, obesity, and smoking [37,38].

\section{Conclusions}

These data suggest that, in the short-term and in a small specific population, plant-based diets are not detrimental to bone in young adults. Moreover, diet prescriptions for bone health may vary among diet groups: increased fruit and vegetable intake for individuals with high meat intakes and increased plant protein intake for individuals who follow a vegetarian diet plan.

\section{Acknowledgments}

The authors thank Ginger Hook, R.N. and Cassandra Smith, R.T. for technical assistance and Lindsay Obermeyer, M.S., R.D. for diet recalls and analyses. 


\section{Author Contributions}

J.K. and C.J. conceived and designed the study; J.K., S.W., and I.A. conducted the study; J.K., S.W., and C.J. analyzed data; J.K., and C.J. wrote the final paper.

\section{Conflicts of Interest}

The authors declare no conflict of interest.

\section{References}

1. Newport, F. In U.S., 5\% Consider Themselves Vegetarians. Available online: http://www.gallup. com/poll/156215/consider-themselves-vegetarians.aspx (accessed on 3 February 2015).

2. Dewell, A.; Weidner, G.; Sumner, M.D.; Chi, C.S.; Ornish, D. A Very-Low-Fat vegan diet increases intake of protective dietary factors and decreases intake of pathogenic dietary factors. J. Am. Diet. Assoc. 2008, 108, 347-356.

3. Key, T.; Appleby, P.; Spencer, E.; Travis, R.; Allen, N.; Thorogood, M.; Mann, J.I. Cancer incidence in British vegetarians. Br. J. Cancer 2009, 101, 192-197.

4. New, S.A. Do vegetarians have a normal bone mass? Osteoporosis Int. 2004, 15, 679-688.

5. Craig, W.J. Nutrition concerns and health effects of vegetarian diets. Nutr. Clin. Pract. 2010, 25, 613-620.

6. Tucker, K.L. Vegetarian diets and bone status. Am. J. Clin. Nutr. 2014, 100, 329S-335S.

7. Deriemaeker, P.; Aerenhouts, D.; Hebbelinck, M.; Clarys, P. Nutrient based estimation of acid-base balance in vegetarians and non-vegetarians. Plant Foods Hum. Nutr. 2010, 65, 77-82.

8. Burckhardt, P. The effect of the alkali load of mineral water on bone metabolism: Interventional studies. J. Nutr. 2008, 138, 435S-437S.

9. Wynn, E.; Krieg, M.A.; Lanham-New, S.A.; Burckhardt, P. Postgraduate symposium: Positive influence of nutritional alkalinity on bone health. Proc. Nutr. Soc. 2010, 69, 166-173.

10. Thorpe, D.L.; Knulsen, S.F.; Beeson, W.L.; Rajaram, S.; Fraser, G.E. Effects of meat consumption and vegetarian diet on risk of wrist fracture over 25 years in a cohort of peri- and postmenopausal women. Public Health Nutr. 2008, 11, 564-572.

11. Ho-Pham, L.T.; Vu, B.Q.; Lai, T.Q.; Nguyen, N.D.; Nguyen, T.V. Vegetarianism, bone loss, fracture and vitamin D: A longitudinal study in Asian vegans and non-vegans. Eur. J. Clin. Nutr. 2012, 66, 75-82.

12. Ho-Pham, L.T.; Nguyen, N.D.; Nguyen, T.V. Effect of vegetarian diets on bone mineral density: A Bayesian meta-analysis. Am. J. Clin. Nutr. 2009, 90, 943-950.

13. Godin, G.; Shephard, R.J. A simple method to assess exercise behavior in the community. Can. J. Appl. Sport Sci. 1985, 10, 141-146.

14. Gans, K.M.; Risica, P.M.; Wylie-Rosett, J.; Ross, E.M.; Strolla, L.O.; McMurray, J.; Eaton, C.B. Development and evaluation of the nutrition component of the Rapid Eating and Activity Assessment for Patients (REAP): A new tool for primary care providers. J. Nutr. Educ. Behav. 2006, 38, 286-292. 
15. Remer, T.; Dimitriou, T.; Manz, F. Dietary potential renal acid load and renal net acid excretion in healthy, free-living children and adolescents. Am. J. Clin. Nutr. 2003, 77, 1255-1260.

16. Ahn, H.; Kim, J.M.; Lee, K.; Kim, H.; Jeong, D. Extracellular acidosis accelerates bone resorption by enhancing osteoclast survival, adhesion, and migration. Biochem. Biophys. Res. Commun. 2012, 418, 144-148.

17. Arnett, T.R.; Dempster, D.W. Effect of $\mathrm{pH}$ on bone resorption by rat osteoclasts in vitro. Endocrinology 1986, 119, 119-124.

18. Schürch, M.A.; Rizzoli, R.; Slosman, D.; Vadas, L.; Vergnaud, P.; Bonjour, J.P. Protein supplements increase serum insulin-like growth factor-I levels and attenuate proximal femur bone loss in patients with recent hip fracture. A randomized, double-blind, placebo-controlled trial. Ann. Intern. Med. 1998, 128, 801-809.

19. Bourrin, S.; Ammann, P.; Bonjour, J.P.; Rizzoli, R. Dietary protein restriction lowers plasma insulin-like growth factor I (IGF-I), impairs cortical bone formation, and induces osteoblastic resistance to IGF-I in adult female rats. Endocrinology 2000, 141, 3149-3155.

20. Josse, A.R.; Atkinson, S.A.; Tarnopolsky, M.A.; Phillips, S.M. Diets higher in dairy foods and dietary protein support bone health during diet- and exercise-induced weight loss in overweight and obese premenopausal women. J. Clin. Endocrinol. 2012, 97, 251-260.

21. Nieves, J.W.; Melsop, K.; Curtis, M.; Kelsey, J.L.; Bachrach, L.K.; Greendale, G.; Sowers, M.F.; Sainani, K.L. Nutritional factors that influence change in bone density and stress fracture risk among young female cross-country runners. PMR 2010, 2, 740-750.

22. Remer, T. Influence of diet on acid-base balance. Semin. Dial. 2000, 13, 221-226.

23. Lemann, J.; Bushinsky, D.A.; Hamm, L.L. Bone buffering of acid and base in humans. Am. J. Physiol. Renal. Physiol. 2003, 285, F811-F832.

24. Sebastian, A.; Harris, S.T.; Ottaway, J.H.; Todd, K.M.; Morris, R.C. Improved mineral balance and skeletal metabolism in postmenopausal women treated with potassium bicarbonate. $N$. Engl. J. Med. 1994, 330, 1776-1781.

25. Michaud, D.S.; Troiano, R.P.; Subar, A.F.; Runswick, S.; Bingham, S.; Kipnis, V.; Schatzkin, A. Comparison of estimated renal net acid excretion from dietary intake and body size with urine $\mathrm{pH}$. J. Am. Diet. Assoc. 2003, 103, 1001-1007.

26. Thorpe, M.; Mojtahedi, M.C.; Chapman-Novakofski, K.; McAuley, E.; Evans, E.M. A positive association of lumbar spine bone mineral density with dietary protein is suppressed by a negative association with protein sulfur. J. Nutr. 2008, 138, 80-85.

27. Adeva, M.M.; Souto, G. Diet-induced metabolic acidosis. Clin. Nutr. 2011, 30, 416-421.

28. Jehle, S.; Zanetti, A.; Muser, J.; Hulter, H.N.; Krapf, R. Partial neutralization of the acidogenic Western diet with potassium citrate increases bone mass in postmenopausal women with osteopenia. J. Am. Soc. Nephrol. 2006, 17, 3213-3222.

29. Jehle, S.; Hulter, H.N.; Krapf, R. Effect of potassium citrate on bone density, microarchitecture, and fracture risk in healthy older adults without osteoporosis: A randomized controlled trial. J. Clin. Endocrinol. 2013, 98, 207-217. 
30. Macdonald, H.M.; Black, A.J.; Aucott, L.; Duthie, G.; Duthie, S.; Sandison, R.; Hardcastle, A.C.; Lanham New, S.A.; Fraser, W.D.; Reid, D.M. Effect of potassium citrate supplementation or increased fruit and vegetable intake on bone metabolism in healthy postmenopausal women: A randomized controlled trial. Am. J. Clin. Nutr. 2008, 88, 465-474.

31. Shiraki, M.; Shiraki, Y.; Aoki, C.; Miura, M. Vitamin K2 (menatetrenone) effectively prevents fractures and sustains lumbar bone mineral density in osteoporosis. J. Bone Miner. Res. 2000, 15, 515-521.

32. Gunn, C.A.; Weber, J.L.; Coad, J.; Kruger, M.C. Increasing fruits and vegetables in midlife women: A feasibility study. Nutr. Res. 2013, 33, 543-551.

33. Campbell, W.W.; Barton, M.L.; Cyr-Campbell, D.; Davey, S.L.; Beard, J.L.; Parise, G.; Evans, W.J. Effects of an omnivorous diet compared with a lactoovovegetarian diet on resistance-traininginduced changes in body composition and skeletal muscle in older men. Am. J. Clin. Nutr. 1999, 70, 1032-1039.

34. Haub, M.D.; Wells, A.M.; Tarnopolsky, M.A.; Campbell, W.W. Effect of protein source on resistive-training-induced changes in body composition and muscle size in older men. Am. J. Clin. Nutr. 2002, 76, 511-517.

35. Schwalfenberg, G.K. The Alkaline Diet: Is There Evidence That an Alkaline pH Diet Benefits Health? J. Environ. Public Health 2012, 2012, 727630, doi:10.1155/2012/727630.

36. Capolongo, G.; Sakhaee, K.; Pak, C.Y.; Maalouf, N.M. Fasting versus 24-h urine pH in the evaluation of nephrolithiasis. Urol. Res. 2011, 39, 367-372.

37. Lanham-New, S.A. Is "vegetarianism” a serious risk factor for osteoporotic fracture? Am. J. Clin. Nutr. 2009, 90, 910-911.

38. Felson, D.T.; Zhang, Y.; Hannan, M.T.; Anderson, J.J. Effects of weight and body mass index on bone mineral density in men and women: The Framingham study. J. Bone Miner. Res. 1993, 8, 567-573.

(C) 2015 by the authors; licensee MDPI, Basel, Switzerland. This article is an open access article distributed under the terms and conditions of the Creative Commons Attribution license (http://creativecommons.org/licenses/by/4.0/). 